\title{
Squeezed Thermal Reservoirs as a Resource for a Nanomechanical Engine beyond the Carnot Limit
}

\author{
Jan Klaers, 'Stefan Faelt, Atac Imamoglu, and Emre Togan \\ Institute for Quantum Electronics, ETH Zürich, CH-8093 Zürich, Switzerland \\ (Received 25 April 2017; revised manuscript received 25 July 2017; published 13 September 2017) \\ The efficient conversion of thermal energy to mechanical work by a heat engine is an ongoing \\ technological challenge. Since the pioneering work of Carnot, it has been known that the efficiency of heat \\ engines is bounded by a fundamental upper limit - the Carnot limit. Theoretical studies suggest that heat \\ engines may be operated beyond the Carnot limit by exploiting stationary, nonequilibrium reservoirs that \\ are characterized by a temperature as well as further parameters. In a proof-of-principle experiment, we \\ demonstrate that the efficiency of a nanobeam heat engine coupled to squeezed thermal noise is not \\ bounded by the standard Carnot limit. Remarkably, we also show that it is possible to design a cyclic \\ process that allows for extraction of mechanical work from a single squeezed thermal reservoir. Our results \\ demonstrate a qualitatively new regime of nonequilibrium thermodynamics at small scales and provide a \\ new perspective on the design of efficient, highly miniaturized engines.
}

\section{INTRODUCTION}

Advances in microtechnology and nanotechnology allow for testing concepts derived from classical thermodynamics in regimes where the underlying assumptions, such as the thermodynamic limit and thermal equilibrium, no longer hold [1-6]. Extremely miniaturized forms of heat engines, in which the working medium is represented by a single particle, have revealed a fluctuation-dominated regime in the conversion of heat to work far away from the thermodynamic limit [7-11]. In the future, the exploitation of novel sources of work could have a large impact on the design of efficient and powerful engines at the microscale and nanoscale. By employing nonequilibrium reservoirs, it is expected that the efficiency of work generation can surpass standard thermodynamic bounds, as has been theoretically suggested for quantum coherent [12], quantum correlated [13,14], quantum-measurement-induced [15-17], and squeezed thermal reservoirs [18-23]. The realization of such engines not only extends our knowledge of finite-size, nonequilibrium, and quantum effects in thermodynamics, but could also lead to important applications in nanotechnology and in the life sciences [24].

In optics, the electric field of a monochromatic wave can be decomposed into two quadrature components that vary as $\cos \omega t$ and $\sin \omega t$, respectively. For coherent states such as laser light, the uncertainties in the two quadratures are

\footnotetext{
*jklaers@phys.ethz.ch
}

Published by the American Physical Society under the terms of the Creative Commons Attribution 4.0 International license. Further distribution of this work must maintain attribution to the author(s) and the published article's title, journal citation, and DOI. equal and follow the lower bound of Heisenberg's uncertainty relation. By contrast, squeezed states of light have reduced fluctuations in one (squeezed) quadrature at the cost of enhanced fluctuations in the other (antisqueezed) quadrature, allowing for optical measurements with reduced quantum noise [25,26]. Squeezed states are, however, restricted neither to electromagnetic waves nor to minimum uncertainty states. For example, a mechanical oscillator may be prepared in a squeezed thermal state [27-29] by a periodic modulation of the spring constant [30]. This results in a state with reduced thermal fluctuations in one quadrature (e.g., momentum) and enhanced thermal fluctuations in the other quadrature (e.g., position) compared to the expected level of fluctuations at the given temperature. In the context of heat engines, a theoretical work by Roßnagel et al. [19] suggests that squeezed thermal states may be used as an additional resource to overcome the standard Carnot limit [31]. Because of the nonequilibrium nature of squeezed thermal reservoirs, this result does not violate the second law of thermodynamics. In our work, we present a physical realization of such an engine with a working medium consisting of a vibrating nanobeam that is driven by squeezed electronic noise to perform work beyond the standard Carnot limit. Furthermore, we demonstrate that by a phase-selective coupling to the squeezed or antisqueezed quadrature, work can be extracted even from a single squeezed reservoir, which is not possible with a standard thermal reservoir [12,21,32].

\section{NANOBEAM HEAT ENGINE}

Figure 1(a) shows a sketch of the experiments: The working medium of our heat engine consists of a single 

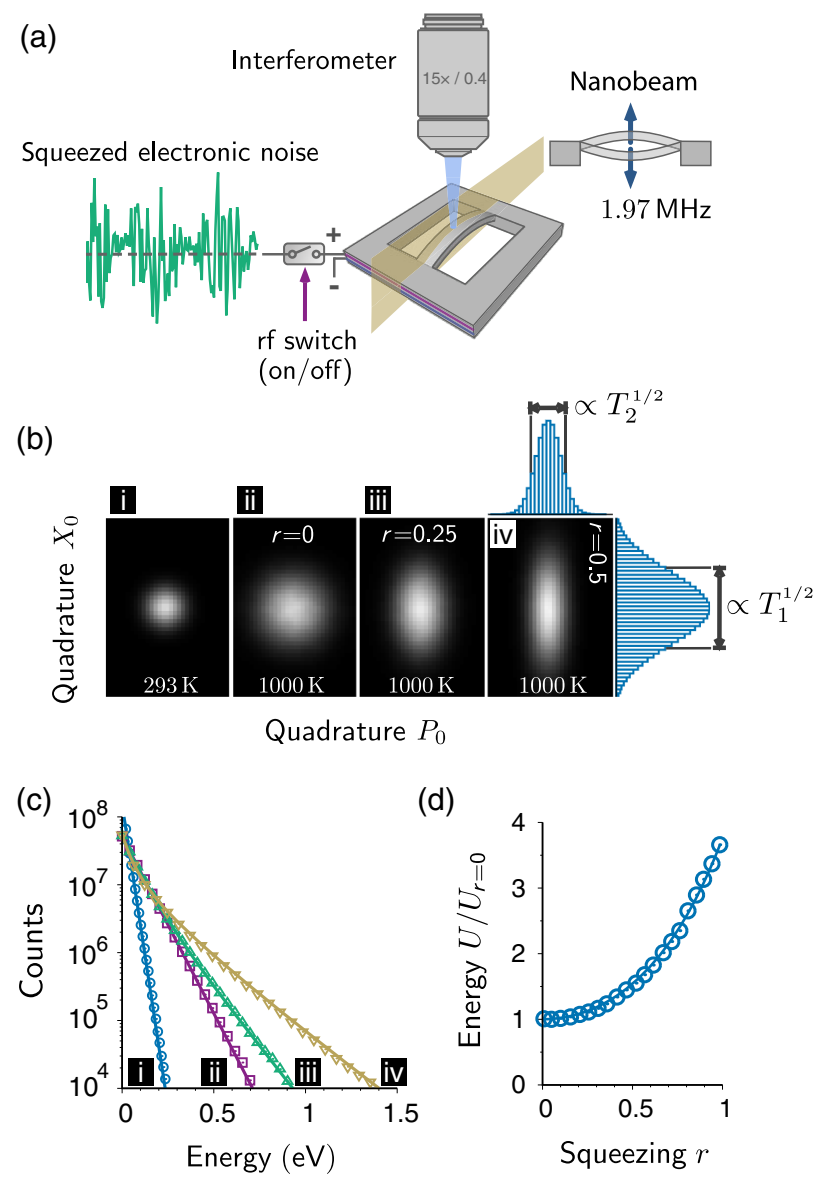

FIG. 1. Nanobeam heat engine. (a) Doubly clamped nanobeam piezoelectrically coupled to squeezed electronic noise. (b) Phasespace density of the nanobeam motion (in rotating frame) (i) when no additional noise is applied and (ii)-(iv) when (squeezed) noise is applied. The fluctuations in the position and momentum quadrature are characterized by two temperatures $T_{1,2}$ proportional to the variance of the line integrated probability densities. The temperatures of the purely thermal states are $T=293 \mathrm{~K}$ for (i) and $T=1000 \mathrm{~K}$ for (ii). The effective temperature of the squeezed thermal states is $T=\sqrt{T_{1} T_{2}} \simeq 1000 \mathrm{~K}$ for (iii) and (iv). The squeezing parameter is $r=0$ for (i) and (ii) and $r=\ln \left(T_{1} / T_{2}\right) / 4=0.25,0.5$ for (iii) and (iv). (c) Measured energy histogram (symbols) of the nanobeam in a squeezed thermal state. The solid lines show the theoretically expected distributions. Experimental parameters as in (b). (d) Energy of the nanobeam (circles) as a function of the squeezing parameter $r$. The energy scale is normalized to the energy at vanishing squeezing $U_{r=0}=k_{B} T$. The solid line corresponds to the theoretical expectation $U / U_{r=0}=1+2 \sinh ^{2} r$. Statistical errors (SEM) are smaller than the symbol size for all data points.

harmonic oscillator given by the fundamental flexural mode of a doubly clamped (GaAs) nanobeam structure with eigenfrequency $\nu=\omega / 2 \pi=1.97 \mathrm{MHz}$, quality factor of order $Q \simeq 10^{3}$ at room temperature, and under vacuum conditions ( $p \simeq 10^{-4}$ mbar). The beam has a length of $18.8 \mu \mathrm{m}$, width of $2 \mu \mathrm{m}$, thickness of $270 \mathrm{~nm}$, and is fabricated using conventional nanostructuring techniques such as electron beam lithography and selective etching. In the growth direction the beam contains two doped layers, which allow us to apply electric fields across the beam material. These electric fields lead to forces being applied to the beam structure due to the piezoelectricity of the employed gallium arsenide material. When driven by a noisy waveform, this creates a random force that allows us to mimic an engineered thermal environment for the beam structure. The waveform is synthesized from two independent white noise signals $\xi_{1,2}(t)$ that are mixed with sine and cosine components of a phase reference at frequency $\nu$ leading to a stochastic force $f(t)=a_{0}\left[e^{+\tilde{r}} \xi_{1}(t) \cos (\omega t)+\right.$

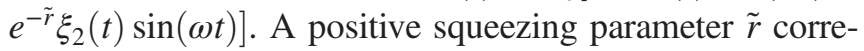
sponds to an amplified cosine and attenuated sine component at frequency $\nu$ in the thermal bath, while the overall strength of the noise can be controlled by the amplitude $a_{0}$.

To demonstrate the generation of squeezed thermal states, we experimentally determine the motional state of the nanobeam by recording the instantaneous position (outof-plane displacement) of the beam via Mach-Zehnder interferometry [33] and calculating the corresponding phase-space probability distribution; see Fig. 1(b). In the absence of additional noise $\left(a_{0}=0\right)$, the nanobeam motion is fully determined by the residual thermal noise at room temperature; see panel (i) in Fig. 1(b). By increasing the noise amplitude $a_{0}$, the state of the nanobeam can be prepared in a thermal state at higher temperature, $T=1000 \mathrm{~K}$; see panel (ii) in Fig. 1(b). Squeezed noise leads to the expected elliptical phase-space probability distribution; see panels (iii) and (iv) in Fig. 1(b). We emphasize that all results are presented in a position-momentum frame that rotates with frequency $\nu$ with respect to the laboratory frame. The observed probability densities closely follow the theoretically expected Gaussian distribution [27],

$$
\rho\left(x_{0}, p_{0}\right) \propto \exp \left(-\frac{\hbar \omega x_{0}^{2}}{2 k_{B} T_{1}}-\frac{\hbar \omega p_{0}^{2}}{2 k_{B} T_{2}}\right),
$$

where $x_{0}, p_{0}$ are dimensionless position and momentum variables and $T_{1}, T_{2}$ are two temperatures describing the level of fluctuations in the antisqueezed and squeezed quadratures (proportional to the variance of the Gaussian distribution). These temperatures allow us to assign an effective temperature $T$ and squeezing parameter $r$ to the squeezed thermal state via $T_{1,2}=T \exp ( \pm 2 r)$ $[27,29]$. The corresponding energy histograms reveal exponential distributions for cases (i) (circles) and (ii) (boxes), as is expected from purely thermal states [Fig. 1(c)]. The nonexponential decays for parameter sets (iii) (upright triangles) and (iv) (upside-down triangles) demonstrate the nonequilibrium nature of the state of the nanobeam. The solid lines in Fig. 1(c) show the theoretically expected energy distribution $\rho(E) \propto I_{0}[E \sinh (2 r) /$ $\left.k_{B} T\right] \exp \left[-E \cosh (2 r) / k_{B} T\right]$, with $I_{0}$ as the modified Bessel function of order zero [33]. Finally, the mean energy 
$U$ of the nanobeam motion as a function of the squeezing parameter $r$ (at fixed temperature) closely follows the caloric equation of state $U=k_{B} T\left(1+2 \sinh ^{2} r\right)$, as demonstrated in Fig. 1(d).

In our experiment, the eigenfrequency of the fundamental flexural mode can be tuned over a few $100 \mathrm{kHz}$ by applying a dc electrical potential to the nanobeam [33] allowing for a cyclic process with work output. The extraction of work in an engine is normally realized by an increase in volume of a gaseous working medium driving a piston. For a harmonically trapped working medium, such as the one we investigate here, an expansion of the working medium may be realized by a decrease in trapping frequency $\omega$. The latter suggests to define the volume of the system as the inverse trapping frequency $V=\omega^{-1}$; see Ref. [34]. With this, a relation similar to the state functional formulation of the first law of thermodynamics holds [33]:

$$
d U=T_{1} d S_{1}+T_{2} d S_{2}-p d V,
$$

with $S_{1}=-k_{B} \int_{-\infty}^{+\infty} \rho\left(x_{0}\right) \ln \left[\rho\left(x_{0}\right)\right] d x_{0}$ as the entropy of the antisqueezed quadrature and the corresponding definition for $S_{2}$ being obtained by replacing $x_{0}$ with $p_{0}$. The pressure is defined by $p=-(\partial U / \partial V)_{S_{1}, S_{2}}$, which evaluates to $p=U / V$ for the given system. In this way, the term $-p d V$ represents the work associated to a change in volume or trapping frequency, whereas the terms $T_{i} d S_{i}$ with $i=1$, 2 describe heat exchange with the environment.

\section{OTTO CYCLE WITH SQUEEZED THERMAL RESERVOIRS}

In a first line of experiments, we construct an Otto cycle between a hot squeezed thermal reservoir with effective temperature $T_{h}=10000 \mathrm{~K}$ and squeezing factor $r=0.4$ and a cold purely thermal bath at $T_{c}=9500 \mathrm{~K}$ under maximum power condition [19]. The four strokes include an adiabatic compression, isochoric heat addition, adiabatic expansion, and isochoric heat rejection. Contrary to the Carnot process, in which the isochoric steps are replaced by isothermal steps, work output in the Otto cycle is solely provided during the adiabatic strokes. Similar to colloidal heat engines [9], the working medium in our system cannot be fully decoupled from its environment rendering the implementation of adiabatic steps not obvious. Experimentally, it is however feasible to replace the adiabatic steps by isentropic steps. The isentropic steps are implemented by simultaneously varying frequency and temperature such that the ratios $T_{1,2} / \omega$ remain constant, which conserves the entropies $S_{1,2}$ [33]. Figure 2(a) shows frequency, squeezing, and temperature of the nanobeam state throughout one cycle of the engine under quasistatic operation. The cycle time $T_{\text {cycle }}$ amounts to several seconds. Note that the frequency of the applied squeezed noise (not shown) follows the frequency of the mechanical oscillator. The pressure-volume $(p-V)$ and temperatureentropy $(T-S)$ diagrams are shown in Figs. 2(b) and 2(c). The shaded area in the $p-V$ diagram corresponds to a net
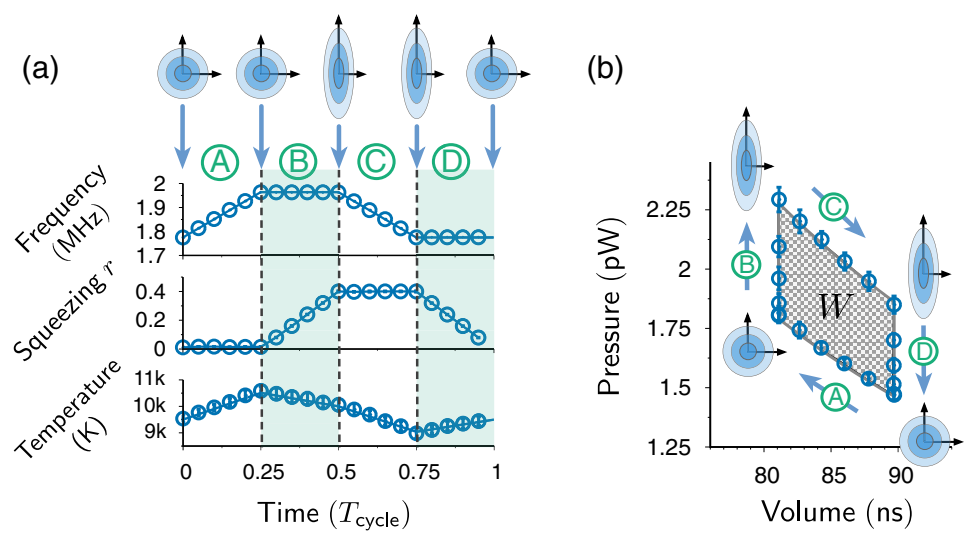

(c)

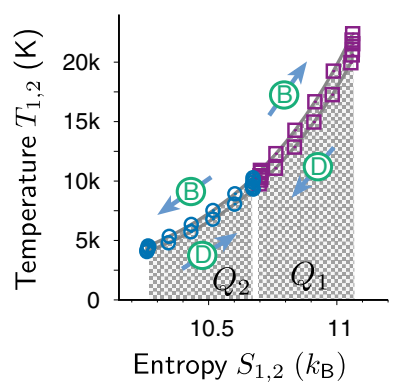

(d)

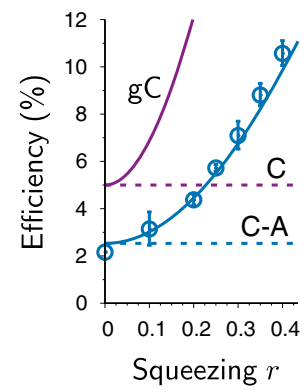

FIG. 2. Otto cycle between a cold thermal and a hot squeezed thermal reservoir. (a) Frequency, squeezing, and temperature of the nanobeam motion throughout one cycle of the engine ( $T_{\text {cycle }}$ amounts to several seconds). The shown protocol implements an Otto cycle at maximum power between a cold thermal reservoir at $T_{c}=9500 \mathrm{~K}$ and a hot squeezed reservoir with effective temperature $T_{h}=10000 \mathrm{~K}$ and squeezing parameter $r=0.4$. The four consecutive strokes include an isentropic compression (A), isochoric heat addition (B), isentropic expansion (C), and isochoric heat rejection (D). (b) Pressure-volume diagram of the Otto cycle. The shaded area corresponds to the total work output $W$ performed by the engine. The solid line represents the theoretically expected behavior. (c) Temperature-entropy diagram of the Otto cycle. $T_{1}\left(T_{2}\right)$ and $S_{1}\left(S_{2}\right)$ denote temperature and entropy of the antisqueezed (squeezed) quadrature. The solid line shows the theoretical expectations. (d) Efficiency of the nanobeam heat engine as a function of the squeezing parameter of the hot reservoir (circles). The solid blue line shows the theoretical expectation $\eta=1-\sqrt{T_{c} / T_{h}} / \cosh (r)$. The measured engine efficiency surpasses the standard Carnot (C) and Curzon-Ahlborn (C-A) efficiency for finite squeezing parameters (dashed horizontal lines), but obeys a generalized Carnot limit (gC) [19,32]. All error bars indicate statistical errors (SEM) except for (d) (see Ref. [33] for details). 
work output of $W \simeq 26 \mathrm{meV}$ performed per cycle. We point out that, presently, this work is neither used to perform a specific task nor stored in some form of potential energy. In the given experiment, the nanobeam performs work against the environment via mechanical strain at the clamping points of the nanobeam, where it excites unconfined mechanical modes that delocalize the work over the system. A known mechanism for work storage in such systems is to attach a second mechanical oscillator with eigenfrequency close to the inverse cycle time to one of the clamping points. The generated work output can then drive a coherent oscillation of this resonator mass [35].

The $T-S$ diagram is composed of two closed curves corresponding to temperature and entropy variation of the squeezed (circles) and antisqueezed quadrature (boxes) within one cycle. Note that the curve for the antisqueezed quadrature is run through clockwise, while the curve for the squeezed quadrature is run through counterclockwise. The net amount of heat consumed from the environment $Q_{h}$ during the hot isochore (B) corresponds to the difference of the two shaded areas, which is $Q_{h}=Q_{1}-Q_{2} \simeq$ $244 \mathrm{meV}$. The efficiency of the cycle finally evaluates to $\eta=W / Q_{h}=(10.6 \pm 0.5) \%$, which is roughly twice the efficiency of a Carnot cycle operating between $T_{h}$ and $T_{c}$ and 4 times the Curzon-Ahlborn efficiency [36]. Figure 2(d) shows the efficiency for varying squeezing parameters of the hot bath. The solid blue line corresponds to the theoretical expectation $\eta=1-\sqrt{T_{c} / T_{h}} / \cosh (r)$, see Ref. [19,33], whereas the two horizontal dashed lines show the standard Carnot (C) and Curzon-Ahlborn (C-A) efficiencies given by $\eta_{\text {Carnot }}=1-T_{c} / T_{h}$ and $\eta_{\mathrm{C}-\mathrm{A}}=$ $1-\sqrt{T_{c} / T_{h}}$, respectively. The latter coincides with the efficiency of the implemented Otto cycle at maximum power for vanishing squeezing [19,33]. These results clearly demonstrate that squeezing, given as a free resource, can be used to increase the work output and efficiency of an engine beyond the standard Carnot limit. The engine operation, however, does obey a generalized Carnot limit (solid line "gC") [19,32].

We emphasize that the costs of providing a squeezed thermal bath are not accounted for in our analysis, which is fully analogous to neglecting the costs (such as heat, work, chemical energy) of providing a hot or cold bath in standard thermodynamics. We also note that the thermodynamic interpretation of the energy flow between system and bath during the hot isochore is controversially discussed in the pertinent literature. Competing notions of heat have been employed, which lead to alternative definitions of the engine efficiency [22,37]. Furthermore, we emphasize that our result does not provide a mean to circumvent the second law of thermodynamics. A combination of two engines, in which one engine synthesizes squeezed heat that is used by another engine to generate work output, would operate at sub-Carnot efficiencies, assuming purely thermal reservoirs as the given resources.

\section{WORK EXTRACTION FROM A SINGLE RESERVOIR}

In a second line of experiments, we eliminate the cold thermal heat bath in the operation of the engine and introduce a phase-selective thermal coupling that allows us to periodically extract work from a single squeezed heat bath. In these measurements, the coupling of the mechanical oscillator to the squeezed electronic noise is periodically switched on and off by means of a rectangular control signal with frequency $2 \nu$ and duty cycle $50 \%$, see top panel in Fig. 3(a), that is applied to a radio-frequency switch. The mean energy of the mechanical oscillator as a function of the relative phase $\Delta$ between the phase of the switching function and the phase of the squeezed bath is shown in the bottom panel of Fig. 3(a) for three different squeezing parameters. For nonvanishing squeezing parameters (boxes and triangles), the measured energy reaches a maximum when the oscillator is coupled to the antisqueezed quadrature of the bath at $\Delta_{\max } \simeq \pi / 2$, whereas an energy minimum is obtained for coupling to the squeezed quadrature at $\Delta_{\min } \simeq 3 \pi / 2$. This phase-selective coupling allows us to extract work from a single squeezed reservoir by a periodic variation of mechanical frequency and phase difference $\Delta$, as shown in the upper two panels of Fig. 3(b) (circles). The four strokes include an "isophasal" compression, during which the phase relation between squeezed noise and periodic coupling is kept fixed, an isochoric heat addition, isophasal expansion, and isochoric heat rejection. Temperature and squeezing of the nanobeam state as a consequence of the applied protocol are shown in the bottom two panels of Fig. 3(b) (circles). Finally, the $p-V$ diagram in Fig. 3(c) clearly reveals a finite amount of work ( $W \simeq 37 \mathrm{meV}$ ) that is extracted from the single squeezed reservoir per cycle (circles). Compared to the case of an unmodulated coupling (switch in "on" position), resulting in a squeezed thermal state with effective temperature $T=10000 \mathrm{~K}$ and $r=0.3$ throughout the cycle [boxes in Figs. 3(b) and 3(c)], the working medium operates at a reduced temperature. The latter originates from the incomplete decoupling of the oscillator from its environment during the "off" phases of the phase-selective coupling. Here, an improved isolation would further increase the extracted work per cycle.

Another possible mechanism for work extraction from a single squeezed reservoir could be the following: A twostroke cyclic process is performed in which the system-bath coupling remains unaltered, but in which the expansion and compression of the working medium is performed in a stroboscopic (and phase-selective) fashion with respect to the phase of the squeezed thermal bath. In this way, the pressure exerted by the antisqueezed quadrature could drive 
(a)
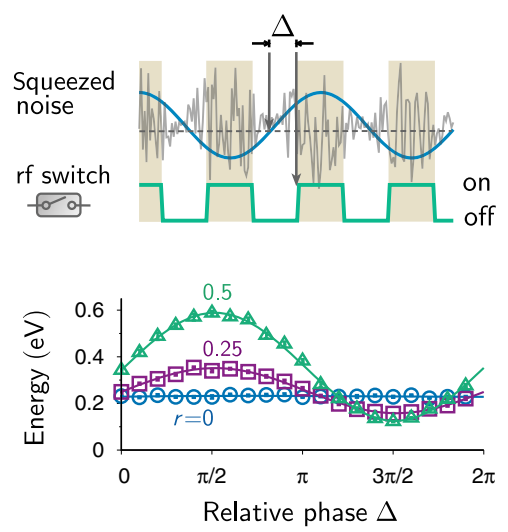

(b)
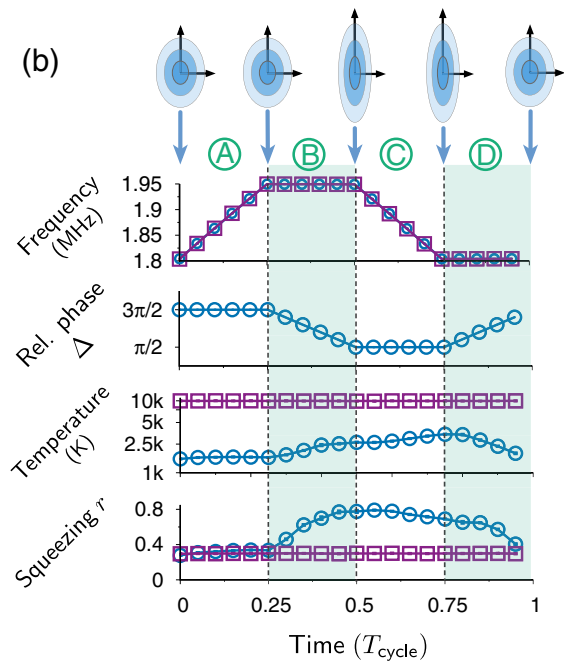

(c)

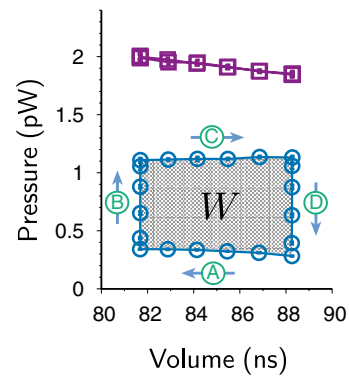

FIG. 3. Work extraction from a single squeezed thermal reservoir. (a) By means of a radio-frequency switch [rf switch in Fig. 1(a)], the coupling between the squeezed electronic noise and the nanobeam is periodically switched on and off with the phase difference between the phase of the squeezed noise and the switching being denoted by $\Delta$ (top). Mean energy of the working medium coupled to a squeezed bath as a function of the relative phase $\Delta$ for three squeezing parameters $r=0,0.25,0.5$ (bottom). The data points are interpolated by harmonic functions with varying amplitude and offset (solid lines). (b) Protocol to extract work from a single squeezed reservoir with effective temperature $T=10000 \mathrm{~K}$ and squeezing parameter $r=0.3$ by periodically varying mechanical eigenfrequency $\nu$ and phase $\Delta$ (circles in upper two graphs). The four strokes include an isophasal compression, during which the phase relation between squeezed noise and periodic coupling is kept fixed, an isochoric heat addition, isophasal expansion, and isochoric heat rejection. Temperature and squeezing of the nanobeam motional state as a consequence of the applied protocol (circles in lower two graphs). The case of an unmodulated coupling is shown with box symbols. Lines between data points are guides to the eye. (c) Pressure-volume diagram revealing a finite amount of work ( $W \simeq 37 \mathrm{meV}$ ) extracted from the single squeezed reservoir per cycle (circles). No work output is observed for unmodulated coupling (boxes). Lines between data points are guides to the eye. Statistical errors (SEM) are smaller than the symbol size for all data points.

the expansion stroke, while the compression stroke would be performed against the pressure exerted by the squeezed quadrature, resulting in a nonzero work output per cycle. This method again would require a fast modulation of a system parameter, in this case the volume change.

\section{CONCLUSIONS}

Single-particle heat machines provide an excellent platform to test theoretical advances in and our understanding of thermodynamics at the microscale and nanoscale. In this work, a minimalist heat engine is realized that takes advantage of squeezed heat to outperform conventional heat engines. The nonequilibrium nature of these reservoirs permits work extraction from a single reservoir and engine efficiencies unbounded by the standard Carnot limit. A major open question remains whether highly miniaturized heat engines, such as the one we report here, will be able to use the extracted work to accomplish mesoscopic tasks as transporting particles [38] or manipulating biological matter [39]. Squeezed thermal noise may naturally arise in nonequilibrium environments with temporally or spatially modulated temperature profiles and could be used to fuel such devices.

\section{ACKNOWLEDGMENTS}

This work is supported by Swiss National Science Foundation (SNSF) through the National Centre of Competence in Research-Quantum Science and Technology (NCCR QSIT), Contract No. 51NF40-160591.

[1] J. Liphardt, S. Dumont, S. B. Smith, I. Tinoco, and C. Bustamante, Equilibrium Information from Nonequilibrium Measurements in an Experimental Test of Jarzynski's Equality, Science 296, 1832 (2002).

[2] S. Toyabe, T. Sagawa, M. Ueda, E. Muneyuki, and M. Sano, Experimental Demonstration of Information-to-Energy Conversion and Validation of the Generalized Jarzynski Equality, Nat. Phys. 6, 988 (2010).

[3] A. Bérut, A. Arakelyan, A. Petrosyan, S. Ciliberto, R. Dillenschneider, and E. Lutz, Experimental Verification of Landauer's Principle Linking Information and Thermodynamics, Nature (London) 483, 187 (2012).

[4] S. Trotzky, Y. A. Chen, A. Flesch, I. P. McCulloch, U. Schollwöck, J. Eisert, and I. Bloch, Probing the Relaxation towards Equilibrium in an Isolated Strongly Correlated One-Dimensional Bose Gas, Nat. Phys. 8, 325 (2012). 
[5] É. Roldán, I. A. Martínez, J. M. R. Parrondo, and D. Petrov, Universal Features in the Energetics of Symmetry Breaking, Nat. Phys. 10, 457 (2014).

[6] For a recent review, see S. Vinjanampathy and J. Anders, Quantum Thermodynamics, Contemp. Phys. 57, 545 (2016).

[7] V. Blickle and C. Bechinger, Realization of a MicrometreSized Stochastic Heat Engine, Nat. Phys. 8, 143 (2012).

[8] J. V. Koski, V. F. Maisi, J. P. Pekola, and D. V. Averin, Experimental Realization of a Szilard Engine with a Single Electron, Proc. Natl. Acad. Sci. U.S.A. 111, 13786 (2014).

[9] I. A. Martínez, É. Roldán, L. Dinis, D. Petrov, J. M. Parrondo, and R. A. Rica, Brownian Carnot Engine, Nat. Phys. 12, 67 (2016).

[10] J. Roßnagel, S. T. Dawkins, K. N. Tolazzi, O. Abah, E. Lutz, F. Schmidt-Kaler, and K. A. Singer, A Single-Atom Heat Engine, Science 352, 325 (2016).

[11] S. Krishnamurthy, S. Ghosh, D. Chatterji, R. Ganapathy, and A. K. Sood, A Micrometre-Sized Heat Engine Operating between Bacterial Reservoirs, Nat. Phys. 12, 1134 (2016).

[12] M. O. Scully, M. S. Zubairy, G. S. Agarwal, and H. Walther, Extracting Work from a Single Heat Bath via Vanishing Quantum Coherence, Science 299, 862 (2003).

[13] R. Dillenschneider and E. Lutz, Energetics of Quantum Correlations, Europhys. Lett. 88, 50003 (2009).

[14] M. Perarnau-Llobet, K. V. Hovhannisyan, M. Huber, P. Skrzypczyk, N. Brunner, and A. Acín, Extractable Work from Correlations, Phys. Rev. X 5, 041011 (2015).

[15] J. Yi, P. Talkner, and Y.W. Kim, Single-Temperature Quantum Engine without Feedback Control, Phys. Rev. E 96, 022108 (2017).

[16] C. Elouard, D. Herrera-Martí, B. Huard, and A. Aufféves, Extracting Work from Quantum Measurement in Maxwell Demon Engines, Phys. Rev. Lett. 118, 260603 (2017).

[17] K. Abdelkhalek, Y. Nakata, and D. Reeb, Fundamental Energy Cost for Quantum Measurement, arXiv: 1609.06981.

[18] X. L. Huang, T. Wang, and X. X. Yi, Effects of Reservoir Squeezing on Quantum Systems and Work Extraction, Phys. Rev. E 86, 051105 (2012).

[19] J. Rossnagel, O. Abah, F. Schmidt-Kaler, K. Singer, and E. Lutz, Nanoscale Heat Engine beyond the Carnot Limit, Phys. Rev. Lett. 112, 030602 (2014).

[20] L. A. Correa, J. P. Palao, D. Alonso, and G. Adesso, Quantum-Enhanced Absorption Refrigerators, Sci. Rep. 4, 3949 (2014).

[21] G. Manzano, F. Galve, R. Zambrini, and J. M. R. Parrondo, Entropy Production and Thermodynamic Power of the Squeezed Thermal Reservoir, Phys. Rev. E 93, 052120 (2016).
[22] W. Niedenzu, D. Gelbwaser-Klimovsky, A. G. Kofman, and G. Kurizki, On the Operation of Machines Powered by Quantum Non-Thermal Baths, New J. Phys. 18, 083012 (2016).

[23] W. Niedenzu, V. Mukherjee, A. Ghosh, A. G. Kofman, and G. Kurizki, Universal Thermodynamic Limit of Quantum Engine Efficiency, arXiv:1703.02911.

[24] T. E. Mallouk and A. Sen, Powering Nanorobots, Sci. Am. 300, 72 (2009).

[25] J. Abadie et al. (LIGO Scientific Collaboration), A Gravitational Wave Observatory Operating beyond the Quantum Shot-Noise Limit, Nat. Phys. 7, 962 (2011).

[26] F. Wolfgramm, A. Cere, F. A. Beduini, A. Predojević, M. Koschorreck, and M. W. Mitchell, Squeezed-Light Optical Magnetometry, Phys. Rev. Lett. 105, 053601 (2010).

[27] H. Fearn and M. J. Collett, Representations of Squeezed States with Thermal Noise, J. Mod. Opt. 35, 553 (1988).

[28] M. S. Kim, F. A. M. de Oliveira, and P. L. Knight, Properties of Squeezed Number States and Squeezed Thermal States, Phys. Rev. A 40, 2494 (1989).

[29] R. R. Tucci, Entropy of a Harmonic Oscillator in Contact with a Squeezed Reservoir, Int. J. Mod. Phys. B 05, 545 (1991).

[30] D. Rugar and P. Grütter, Mechanical Parametric Amplification and Thermomechanical Noise Squeezing, Phys. Rev. Lett. 67, 699 (1991).

[31] S. Carnot, Réflexions sur la Puissance Motrice du feu et sur les Machines Propres a Développer Cette Puissance (Bachelier, Paris, 1824).

[32] O. Abah and E. Lutz, Efficiency of Heat Engines Coupled to Non-Equilibrium Reservoirs, Europhys. Lett. 106, 20001 (2014).

[33] See Supplemental Material at http://link.aps.org/ supplemental/10.1103/PhysRevX.7.031044 for further experimental and theoretical details.

[34] V. Romero-Rochcín, Equation of State of an Interacting Bose Gas Confined by a Harmonic Trap: The Role of the “Harmonic” Pressure, Phys. Rev. Lett. 94, 130601 (2005).

[35] P. G. Steeneken, K. le Phan, M. J. Goossens, G. E. J. Koops, G. J. A. M. Brom, C. van der Avoort, and J. T. M. van Beek, Piezoresistive Heat Engine and Refrigerator, Nat. Phys. 7, 354 (2011).

[36] F. L. Curzon and B. Ahlborn, Efficiency of a Carnot Engine at Maximum Power Output, Am. J. Phys. 43, 22 (1975).

[37] B. Gardas and S. Deffner, Thermodynamic Universality of Quantum Carnot Engines, Phys. Rev. E 92, 042126 (2015).

[38] P. Hänggi and F. Marchesoni, Artificial Brownian Motors: Controlling Transport on the Nanoscale, Rev. Mod. Phys. 81, 387 (2009).

[39] S. M. Douglas, I. Bachelet, and G. M. Church, A LogicGated Nanorobot for Targeted Transport of Molecular Payloads, Science 335, 831 (2012). 